\title{
MESURE DE LA CROISSANCE INDIVIDUELLE DES BRINS DE LAINE A L'AIDE DE RADIOCYSTINE
}

\author{
PAR
}

\section{J. ROUGEOT}

Station de Physiologie Animale; Jouy-en-Josas (S.-et-O.).

Jusqu'à présent il était impossible de suivre individuellement la croissance en longueur des fibres de la toison du Mouton, faute de pouvoir inscrire simultanément sur un certain nombre d'entre elles des repères suffisamment précis, à une date bien définie.

Nous avons résolu ce problème en injectant périodiquement dans la peau, toujours au même point, une très faible dose de cystine marquée au ${ }^{35} \mathrm{~S}$ et en révélant sur les fibres, par autoradiographie, les points de radioactivité correspondant aux dates des injections.

La radiocystine ${ }^{35} \mathrm{~S}$ présente $\dot{a}$ priori des avantages dont certains ont été mis en évidence par les travaux de BERN, HARKNEss et BI,AIR (I955), et surtout par ceux de RYDER (I957, I958a, I958b) : I) cet acide aminé participe à la synthèse de la kératine dans des proportions importantes, soit, en moyenne, Io p. IOO ; 2) on le retrouve dans le follicule, quelques minutes après l'injection, à un niveau très précis, juste avant la zone dite de prékératinisation ; 3) par la suite, la cystine est rapidement épuisée puisqu'on n'en trouve que des traces au bout de trois semaines ; 4) la période $d u^{35}$ S étant de 87 jours, est suffisamment longue pour obtenir plusieurs mois après l'injection une image par autoradiographie.

Nous avons en outre établi les faits suivants : I) après une injection souscutanée de io y.c, les brins de laine se trouvant dans un rayon de plus de $2 \mathrm{~cm}$ du point d'injection, ne présentent pas d'activité décelable au compteur ; 2) la partie des brins de laine, repoussant après un rasage effectué trois semaines après une injection sous-cutanée de Io :.c, ne présentent plus de radioactivité décelable au compteur ; 3) 6 injections sous-cutanées de Io $\mu \mathrm{c}$, faites à intervalles de 3 semaines, exactement au même point, ne provoquent aucune lésion visible ; 4) même avec une injection de I w.c, on obtient, sur les brins étendus sur lame et enrobés d'émulsion coulable, des points d'une netteté suffisante pour les mesures 4 mois après l'injection et après 15 jours d'exposition. La précisions du marquage peut être estimée à IoO $\mu$, soit environ $I 2$ heures de croissance pour les brins courts.

Il était donc possible, par les injections de radiocystine ${ }^{35} \mathrm{~S}$, espacées de 
3 semaines, de révéler sur les brins de laine, par autoradiographie, une succession de points datés avec précision.

\section{Distances en mm entre 2 points successifs} de radio-activité révélés sur les brins de laine

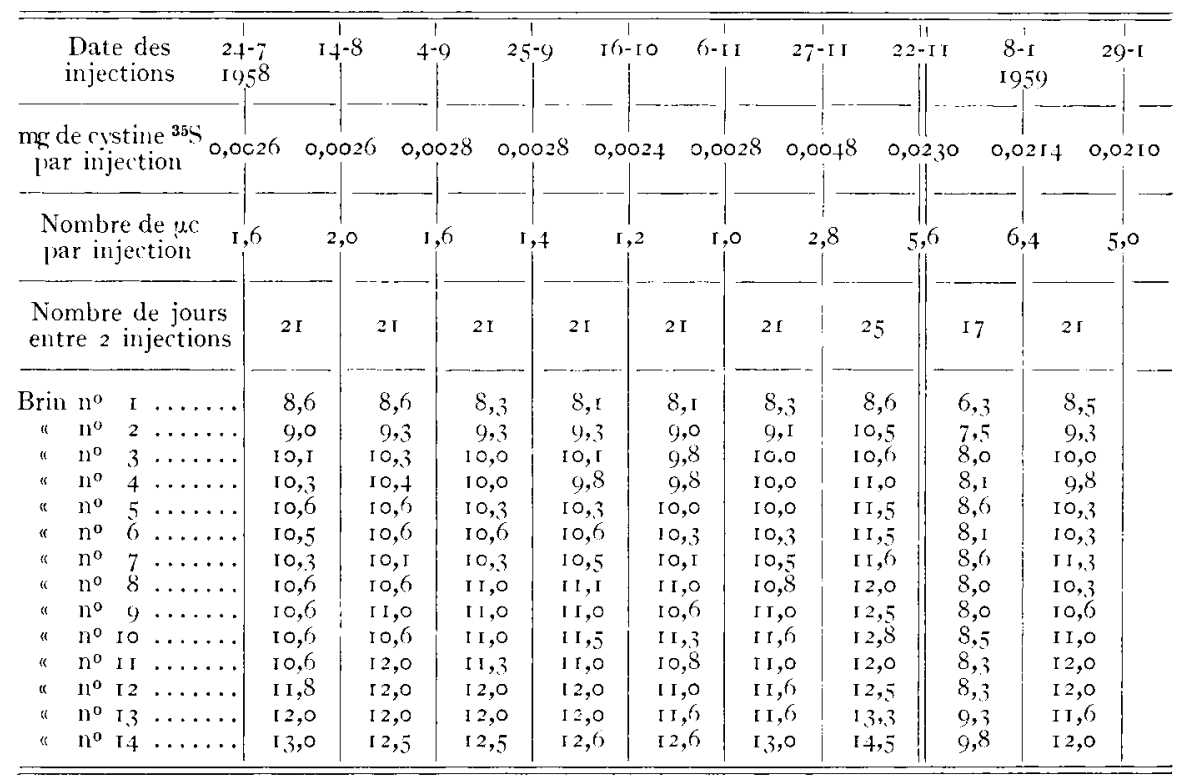

Nous avons entrepris de suivre, avec cette méthode, la croissance annuelle des brins de laine dans les toisons homotriches de quatre Brebis Ile-de-France adultes, maintenues en état physiologique stable (régime alimentaire constant, pas de gestation ni (le lactation, pas de croissance).

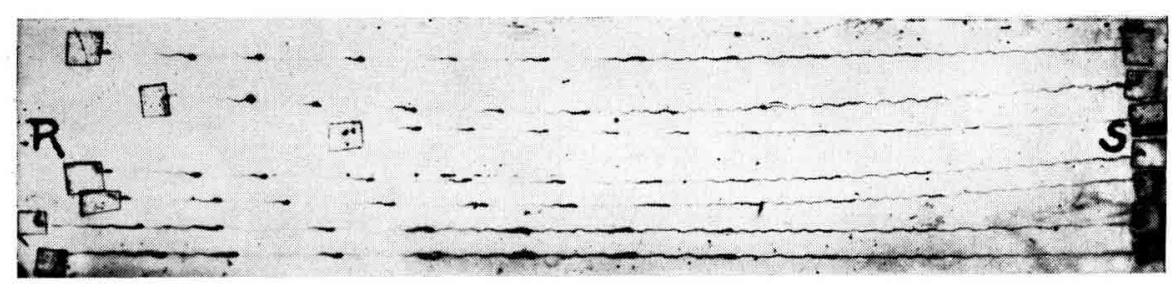

Autoradiographie des brins de laine ( $\mathrm{K}$ : racine ; $\mathrm{S}$ : sommet).

Au bout de six mois, un prélèvement effectué sur une des Brebis montra effectivement que chacune des dix injections faites à intervalles de 2 I jours (de 25 jours après la $7^{\mathrm{e}}$, et de $\mathrm{I} 7$ jours après le $8 \mathrm{e}$ ) avait marqué chaque brin de laine de la zone traitée par un point de radioactivité nettement révélé dans l'émulsion après 53 jours d'exposition (photographie ci-dessus).

Les intervalles entre les points furent mesurés par projection microscopique au curvimètre à $2 /$ Io de mm près, en suivant les ondulations de la laine : 
le tableau ci-joint montre que la croissance en longueur de chaque brin s'est faite à une vitesse très sensiblement constante, pour chacun et en particulier qu'elle n'a pas reflété les variations des doses de cystine injectée.

Ainsi, avec cette méthode, il est possible de stivive dans la toison, avec précision, pendant plusieurs mois, la croissance en longueur individuelle des fibres d'une même mèche.

\section{REFERENCES BIBLIOGRAPHIQUES}

Bern (H. A.), Harkness (1. R.) et Bi.air (S. M.). - Proc. nat. Acad. Sci, U. S. A., 51, 55-60., I955.

Ryder (M. L.). - fe Congr. internation. Nutrit., Rés. Comm., Paris, 6o-6I, I 957 .

Ryder (M. L.). - In The biology of hair growth, Acad. Press. I vol., 305-334, I958a.

Ryder (M. L.). - Proc. roy. Soc. Edinb., B, 67, 65-82, $1958 b$. 